\title{
TRAUMATIC DISLOCATION OF THE RADIUS AT THE ELBOW
}

\author{
J. J. Wiley, ${ }^{*}$ John Pegington, $\nmid$ and J. P. Horwich, ${ }^{*}$ Ottawa, Canada \\ From the Departments of Orthopaedic Surgery and Anatomy, University of Ottawa
}

Traumatic dislocation of the radius at the elbow as an isolated lesion is considered a rare injury. Other authors have described the condition, particularly the clinical features including the classification of the types of dislocation, the possible mechanism of injury and the management (Thomas 1929, Stelling and Cote 1956, Travaglini 1962, Vesely 1967, DePalma 1970, Smith 1972). Scant knowledge is available as to the nature of the pathological lesion which permits such an unusual displacement of the radius (Spinner and Kaplan 1970). The purpose of this paper is to report ten cases of this injury, with a study of the relevant anatomical features.

\section{MECHANISM OF INJURY}

From the history it is impossible to be certain of the exact position of the forearm and elbow at the time of injury. It is probable that the elbow is partly outstretched and the forearm pronated (Vesely 1967). Further violence is applied to the pronated forearm in at least one

TABLE I

Relevant Details of Ten Patients

\begin{tabular}{|c|c|c|c|c|}
\hline $\begin{array}{c}\text { Case } \\
\text { number }\end{array}$ & Sex & $\begin{array}{c}\text { Age } \\
\text { (years) }\end{array}$ & $\begin{array}{c}\text { Side } \\
\text { affected }\end{array}$ & $\begin{array}{l}\text { Direction of } \\
\text { dislocation }\end{array}$ \\
\hline 1 & Female & 8 & Left & Anterior \\
\hline 2 & Male & 7 & Left & Lateral \\
\hline 3 & Male & 10 & Right & Anterior \\
\hline 4 & Female & 5 & Right & Lateral \\
\hline 5 & Male & 48 & Left & Lateral \\
\hline 6 & Female & 5 & Left & Lateral \\
\hline 7 & Male & 9 & Left & Lateral \\
\hline 8 & Male & 32 & Right & Lateral \\
\hline 9 & Male & 7 & Left & Anterior \\
\hline 10 & Male & 5 & Left & Anterior \\
\hline
\end{tabular}

of two ways: 1) with the arm held behind the back, the patient then falls backwards on his forearm, producing hyperpronation (Corbett 1931);2) with the affected arm fixed on the ground (as in a one-hand handstand) the patient's body pivots around this extremity (Vesely 1967). In either case the added body weight increases the pronation and applies a varus strain to the elbow. Eventually the annular ligament ruptures laterally or antero-laterally and the radial head escapes from the joint. The dislocated proximal radius may eventually assume a position lateral, anterior, or even postero-lateral to the elbow joint proper. Its final location probably depends on the positioning of the extremity after the injury has occurred.

* Department of Orthopaedics, Ottawa General Hospital.

† Department of Anatomy, Faculty of Medicine, University of Ottawa.

VOL. 56 B, No. 3, AUGuSt 1974 


\section{CLINICAL FEATURES}

The patient, commonly a child, presents with a painful elbow, guarded against any movement. Little if any distortion is apparent. Passive movement may be surprisingly free in flexion and extension, but there is marked resistance to pronation and supination. Depending upon the position of the elbow, the dislocation of the proximal end of the radius may easily be missed on the radiograph (Wang 1967), or even over-diagnosed as a total dislocation of the elbow. It is well to remember that on a lateral radiograph of the normal elbow a line drawn through the long axis of the radius bisects the capitulum.

Reduction is accomplished by gentle supination of the forearm combined with extension of the elbow and, if necessary, direct thumb pressure over the radial head. In one of our cases an unstable reduction was managed by driving a Kirschner wire across the joint from the capitulum to the radial head. The elbow is immobilised in a posterior plaster splint for three weeks and then gently mobilised.

\section{CLINICAL MATERIAL}

Relevant details of the ten patients in the series are shown in Table I. The patients were aged from five to forty-eight years but only two were adults. All of the children were aged ten years or less. The right elbow was involved in only three instances. Six dislocations were lateral and four anterior. All were closed injuries, so there was no opportunity to examine the extent of the soft-tissue injury. There were no complications related to nerves or vessels. Reduction was accomplished in all cases.

Nine patients were available for follow-up examination, the interval from accident to review ranging from seven months to seven years. All nine were free from symptoms and unaware of any limitation of movement. On examination, however, six patients had restriction of flexion or extension ranging from 5 to 20 degrees. Full pronation and supination had been restored in all patients. There was no instance of recurrence of the dislocation.

\section{ILLUSTRATIVE CASE REPORTS}

Case 1-A girl aged eight years sustained an anterior dislocation of the left radius at the elbow in a fall (Fig. 1). Reduction was performed under general anaesthesia. Follow-up examination two years later showed full restoration of elbow movement. There were no symptoms related to the elbow.
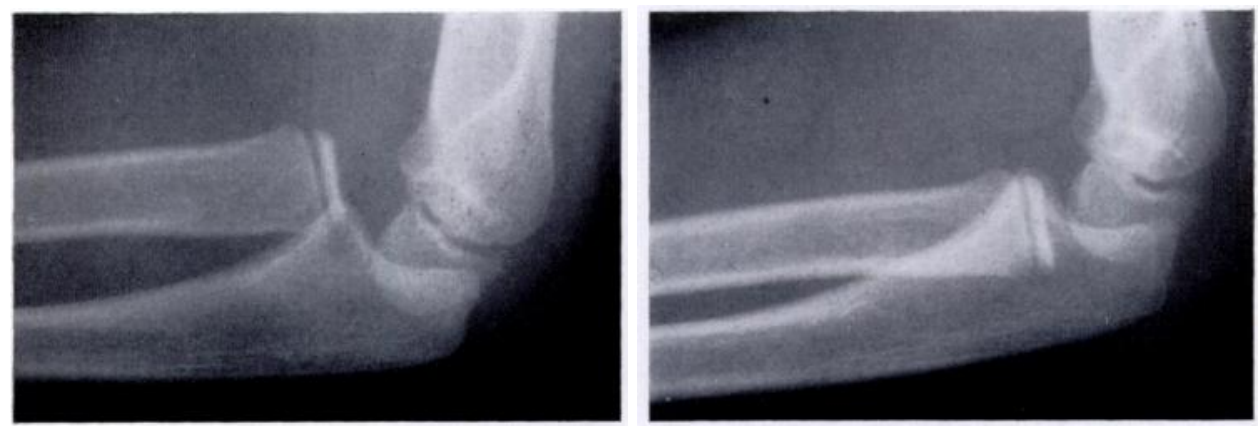

Fig. 1

Case 1-Anterior dislocation of radius at elbow, with normal elbow for comparison (right).

Case 2-A boy aged seven fell from a tree sustaining a lateral dislocation of the left radius (Fig. 2). The injury was initially described as a total dislocation of the elbow. Reduction was performed under general anaesthesia, the radius reducing as the forearm was supinated. After three weeks of immobilisation he was allowed full use of the elbow. Five years later the elbow still lacked 10 degrees of extension, although he had no symptoms. 
Case 3-A boy aged ten sustained an anterior dislocation of the proximal right radius when he fell from a slide (Fig. 3). The dislocation was reduced under general anaesthesia. The elbow was immobilised in a posterior plaster splint for three weeks and then mobilised. Seven years later there were no symptoms from the elbow; the patient was unaware of any limitation of movement although the elbow lacked 10 degrees of extension.

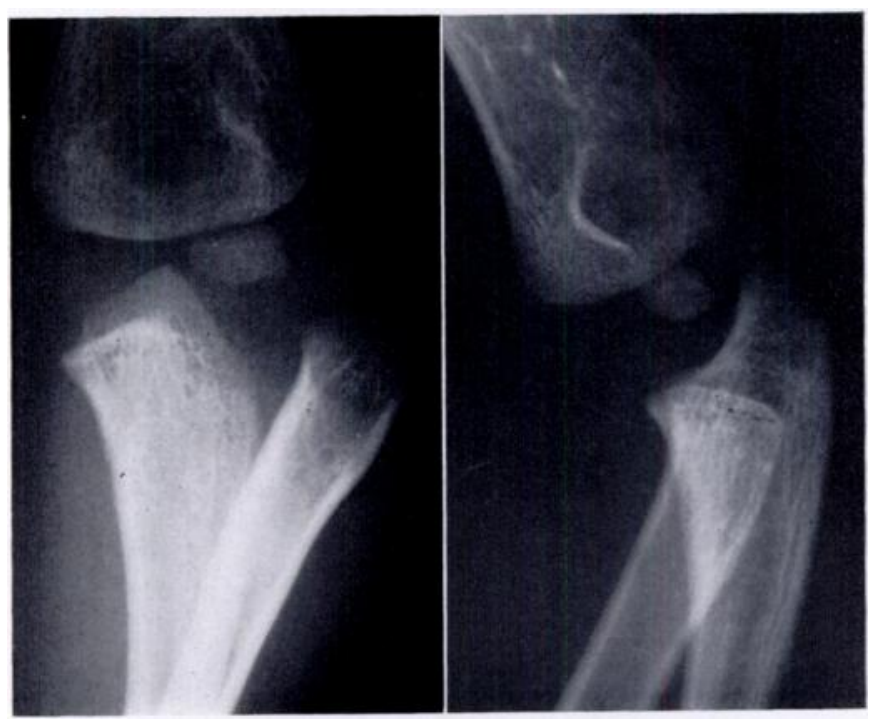

Fig. 2

Case 2-Lateral dislocation.

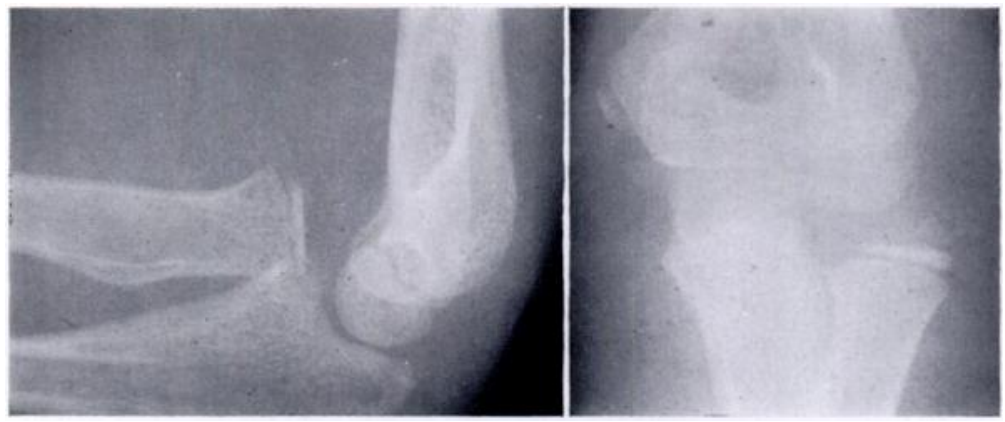

FIG. 3

Case 3-Anterior dislocation.

\section{PATHOLOGICAL ANATOMY}

Lateral dislocation of the radius was repeatedly reproduced in the elbows of corpses by stripping the muscles enclosing the lateral aspect of the elbow, accurately incising the capsule and annular ligament, and applying certain forces to the forearm. With a lateral capsular incision, extended to include the annular ligament, the radial head could be dislocated from its position with forced pronation of the forearm and varus strain at the elbow (Fig. 4). This dislocation included rupture of the quadrate ligament, but no apparent injury to the interosseous membrane. The humero-ulnar joint remained apparently undisturbed.

With the radial head dislocated laterally, supination of the forearm either reduced the radial head to its normal position or directed the head to a seemingly irreducible position posteriorly. Although in none of our patients was dislocation posterior, such dislocation has been described.

vol. 56 B, No. 3, AUgUSt 1974 
Anterior dislocation of the proximal end of the radius could be reproduced with difficulty in cadaveric specimens with the forearm in extreme supination, by completely severing the anterior capsule and annular ligament, and applying force in an anterior direction to the posterior aspect of the radial head. It did not appear that simple over-pull of the biceps tendon could produce such an injury. In a number of specimens tested this type of anterior dislocation could not be reproduced without extensive disruption of the annular ligament and considerable force, in some cases tearing the upper portion of the interosseous membrane.

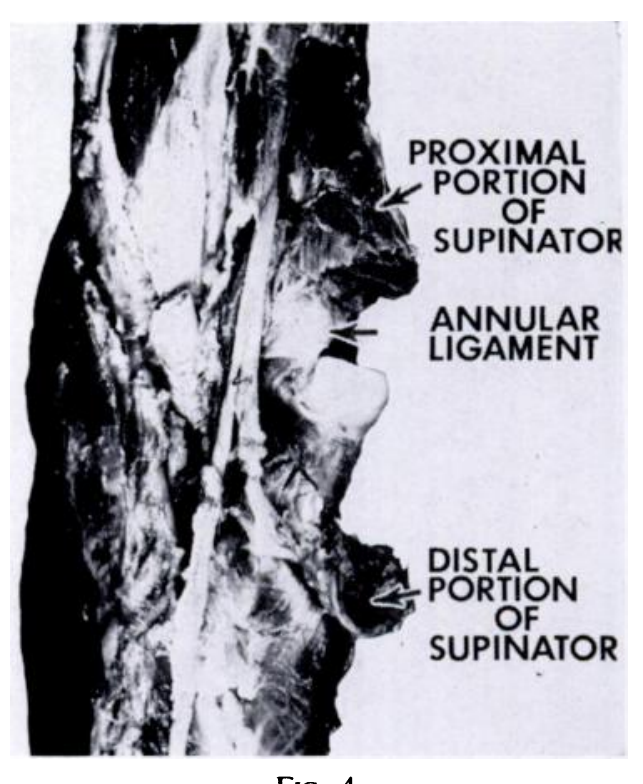

Fig. 4

Cadaveric specimen-the supinator muscle has been severed and retracted. There is lateral dislocation of the radial head. Note the tear of the lateral joint capsule and annular ligament.

Normal anatomy-During pronation and supination several structures maintain the radial head within the radial notch of the ulna, thus stabilising the proximal radio-ulnar joint. These structures include the joint capsule, certain ligaments and the interosseous membrane.

The capsule of the elbow joint extends distally to blend with the strong annular ligament which encircles the head and neck of the radius. The ligament is attached to the anterior and posterior margins of the ulnar notch. Its lowest fibres gain loose attachment to the neck of the radius so that it does not interfere with the rotatory movements of the radial head (Fig. 5).

The capsule is also strengthened on the lateral aspect of the joint by the radial collateral ligament. This thickened part of the capsule is triangular in shape, its apex being attached to the lateral epicondyle and its base to the annular ligament. The anterior and posterior fibres sweep around in front of and behind the joint as far as the borders of the radial notch of the ulna.

The quadrate ligament supports the synovial membrane at the inferior limit of the proximal radio-ulnar joint. This small ligament extends between the radial neck and the inferior border of the radial notch (Fig. 6).

The interosseous membrane is a strong fibrous sheet which stretches between the interosseous borders of the radius and ulna. It has a free upper edge. Its fibres course obliquely downwards from radius to ulna, so allowing for the transmission of forces from the hand proximally into the extremity (Fig. 5). 
Above the upper border of the interosseous membrane is a variable fibrous band stretching from the tuberosity of the ulna to the radius just below its tuberosity. This structure is called the oblique cord. Its obliquity is in the opposite direction to the fibres of the interosseous membrane and it probably represents a portion of the fascia which is sometimes found on the deep surface of the supinator muscle (Martin 1958) (Fig. 5).

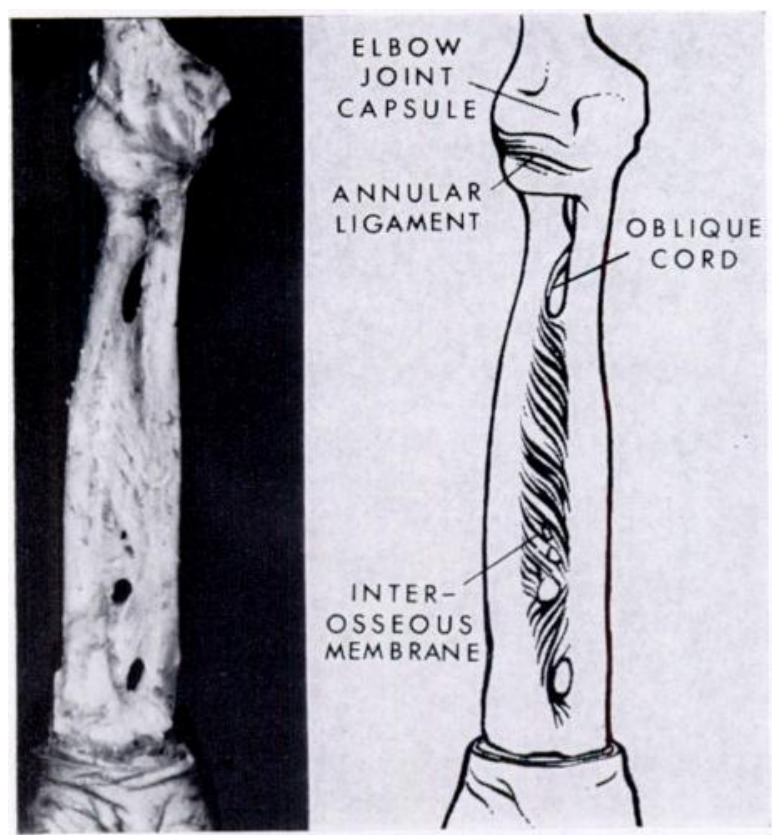

Fig. 5

The stabilising structures which maintain the radio-humeral joint.
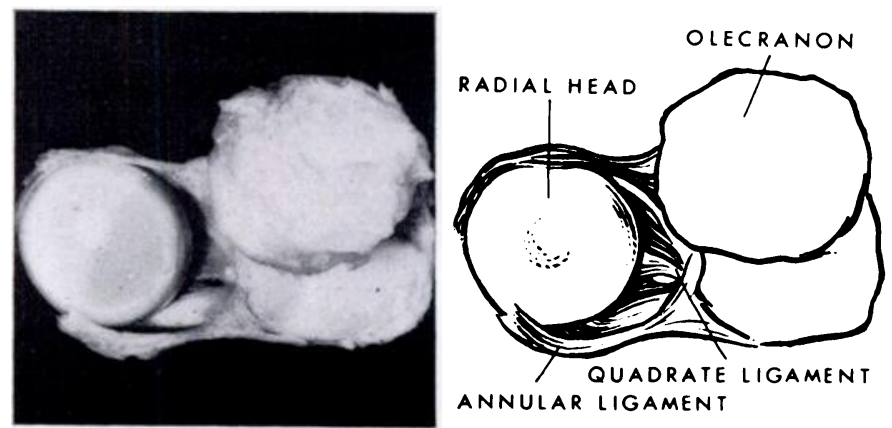

Fig. 6

The quadrate ligament. The annular ligament has been incised laterally. Radio-ulnar laxity is noted with neutral forearm rotation.

The supinator muscle arises behind the elbow from the lateral epicondyle and from the ulna and sweeps around the annular ligament and radius to its radial insertion. It also preserves to some extent the integrity of the region.

The stability of the upper end of the radius was studied on dissected cadaveric specimens. The role of individual stabilising structures-Selective removal of the stabilising structures provided information on the role played by each of them in maintaining the normal position of the radial head during supination and pronation. 
Firstly, a series of specimens was prepared in each of which the interosseous membrane, supinator with its oblique cord, the quadrate ligament and the lateral elbow ligament had all been removed. Thus the only structure left intact to stabilise the upper end of the radius was the annular ligament, which maintained the radial head in its normal snug relationship to the ulna in all positions of the forearm-pronation, neutral and supination.



FIG. 7

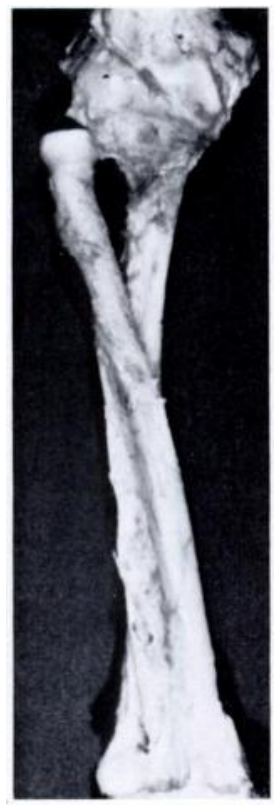

FIG. 8

Figure 7 -The interosseous membrane and the annular ligament are intact. In pronation the membrane is lax. Figure 8-After lateral incision of the annular ligament the lax interosseous membrane allows dislocation of the head of the radius as the forearm is pronated further.

Secondly, specimens were prepared in which the interosseous membrane, supinator muscle with its oblique cord, and the lateral ligament of the elbow had been removed. The annular ligament was divided completely laterally so that the only stabilising structure intact was the quadrate ligament. In the neutral position of the forearm the radial head fitted only loosely into the radial notch (Fig. 6). However, with pronation the posterior fibres of the quadrate ligament became taut, rigidly holding the radial head in the radial notch of the ulna. Similarly, supination provided good stabilisation as the anterior fibres of this ligament tightened (Kaplan 1964). Although this ligament undoubtedly reinforces the stability of the normal proximal radio-ulnar joint, disruption readily occurs if the limits of rotation of the forearm are exceeded.

Thirdly, specimens were prepared with all structures removed except the interosseous membrane and the annular ligament. Pronation and supination produced varying degrees of tension in the membrane. In pronation there was quite remarkable laxity of the membrane because in this position the interosseous borders of radius and ulna are more closely approximated and the membrane is folded between them (Fig. 7). The membrane is moderately taut in the neutral position and quite taut when the forearm is supinated (Fig. 1). With extreme supination some degree of slackening reappears.

The annular ligament of these specimens was then completely incised laterally. When the forearm was pronated the laxity of the interosseous membrane allowed the radial head to be dislocated laterally to such an extent that it slipped off the capitulum (Fig. 8). Such a degree of lateral movement was not possible in supination, the head only being capable of slipping one or two millimetres, but still articulating with the capitulum.

Finally, several specimens were found with a good oblique cord. Pronation of the forearm produced laxity of the band, but supination brought it into full tension. If any reinforcing effect occurs from this small cord it would thus be only in the supinated position (Martin 1958). It should again be emphasised that the oblique cord is not always present.

\section{CONCLUSIONS}

These observations indicate that the most important structure maintaining the normal anatomical position of the upper end of the radius is the annular ligament. The interosseous membrane stabilises the relationship only when the forearm is in the neutral or supinated positions. The oblique cord and the quadrate ligament offer only limited support. The cord, if present, confers some stability in the supinated position, and the ligament in the extremes of pronation and supination. For practical purposes, however, these two structures are 
considered of insufficient strength to influence significantly the forces required to dislocate the radius (Kaplan 1964). From these facts it is deduced that the most likely position of the forearm for lateral dislocation of the radial head would be one of pronation.

\section{SUMMARY}

1. Isolated dislocation of the radius at the elbow occurs most commonly as a pronation injury, associated with slight elbow flexion and a varus strain. Disruption of the radio-ulnar articulation occurs primarily because of tearing of the annular ligament, which is the most important reinforcing structure of this joint. The tensing of the interosseous membrane through neutral into supination, and consequently the approximation of the radius to ulna, supports the recognised supination manoeuvre to reduce such an injury.

2. It is suggested that this injury may be more common than previously appreciated. It may be not diagnosed, it may be over-diagnosed as total dislocation of the elbow, or it may be belatedly diagnosed as a congenital dislocation of the radial head.

\section{REFERENCES}

Corbett, C. H. (1931): Anterior dislocation of the radius and its recurrence. British Journal of Surgery, $19,155-157$.

DePalma, A. F. (1970): The Management of Fractures and Dislocations-An Atlas. Second edition. Volume 1, p. 749. Philadelphia, London, Toronto: W. B. Saunders.

KaPLAN, E. B. (1964): The quadrate ligament of the radio-ulnar joint of the elbow. Bulletin of the Hospital for Joint Diseases, 25, 126-130.

MARTIN, B. F. (1958): The oblique cord of the forearm. Journal of Anatomy, 92, 609-615.

Smith, F. M. (1972): Surgery of the Elbow. Second edition. Philadelphia: W. B. Saunders Company.

SPINNER, M., and Kaplan, E. B. (1970): The quadrate ligament of the elbow-its relationship to the stability of the proximal radio-ulnar joint. Acta orthopaedica Scandinavica, 41, 632-647.

Stelling, F. H., and Cote, R. H. (1956): Traumatic dislocation of head of radius in children. Journal of American Medical Association, 160, 732-736.

Tномаs, T. T. (1929): A contribution to the mechanism of fractures and dislocations in the elbow region. Annals of Surgery, 89, 108-121.

Travaglini, F. (1962): La lussazione traumatica isolata del capitello radiale. Archivo "Putti" di chirurgia degli organi di movimento, 16, 422-441.

VeSELY, D. G. (1967): Isolated traumatic dislocations of the radial head in children. Clinical Orthopaedics and Related Research, 50, 31-36.

Wang, S. K. (1967): Roentgen diagnosis of radial head dislocation. Pacific Medicine and Surgery, 75, 22-25. 